admission (HR $10.5 \mathrm{p}=0.001)$ predicted mortality within 365 days of surgery. The median length of stay was 11 days (0304). TIPS predicted survival (OR 8.3, p=0.019). Median overall survival in the cohort was 64-months.

Conclusions Symptomatic umbilical hernias in patients with cirrhosis can be treated safely with acceptable outcomes in a specialist liver transplant centre, however this remains a high risk intervention. We attribute our outcomes to a multidisciplinary approach to management of patients with ESLD and an umbilical hernia. TIPS may improve short-term outcomes but further prospective trials are warranted.

\section{PWE-40 IMPROVING CARE OF PATIENTS WITH HEREDITARY HAEMOCHROMATOSIS (HH) - A REVIEW FROM A LARGE DGH}

${ }^{1}$ Abhishek Gairola*, ${ }^{2}$ Deepak Kejariwal. 'Northumbria Healthcare NHS Foundation Trust, Newcastle, UK; ${ }^{2}$ County Durham And Darlington NHS Foundation Trust, Durham, UK

\subsection{6/gutjnl-2021-BSG.230}

Introduction The joint BSG/BSH guidance on management of $\mathrm{HH}$ sets out clear criteria for the diagnosis and management of $\mathrm{HH}$ (Fitzsimons et al, 2018). Historically, patients with $\mathrm{HH}$ have been managed by both haematologists and gastroenterologists, mostly based on initial referral from primary care. Our aim was to assess adherence to the initial treatment pathway, and guidance on referral to gastroenterology.

Methods This was a retrospective study. A patient list was generated in June 2020 from patients with $\mathrm{HH}$ listed to attend Day Unit for regular venesection. Review of the electronic patient record was undertaken for each patient to obtain demographics, biochemical parameters at diagnosis, initial imaging, and subsequent management.

Results One hundred and twenty patient records were analysed. Seven records were excluded due to lack of data, leading to a final sample size of 113 . Eighty were male, 33 were female with an average age of 59.3 (51.2 at diagnosis). Fiftyone percent of patients presented initially to haematology, with the remainder presenting to gastroenterology. Sixty-two percent of patients underwent weekly venesection and $70 \%$ had a target maintenance serum ferritin level of $<50 \mathrm{mcg} / \mathrm{L}$. Of the cohort presenting to haematology, 38\% were not referred to gastroenterology despite meeting referral criteria. Of the 12 patients referred according to guidance, 5 underwent fibrosis assessment. Overall, 58 patients were identified who would have benefited from fibrosis assessment. Seventyeight patients underwent ultrasound scans of the abdomen (with an average of 3 scans per patient) compared to 18 patients undergoing FibroScan.

Conclusions In general, adherence to the initial treatment pathway was variable - with the acknowledgement that in clinical practice, several factors lead to poor concordance with weekly venesection. Serum ferritin measurement was used to guide treatment in all cases. In all cases, upon confirming diagnosis, reference was made to 'regular venesection'. Initial target ferritin was never, or very rarely, set as low as 20-30 $\mathrm{mcg} / \mathrm{L}$. The initial target in the Trust was set as less than $50 \mathrm{mcg} / \mathrm{L}$.

For patients presenting initially to haematology there was inconsistency in making referrals to gastroenterology. A proportion of this cohort would benefit from fibrosis assessment based on current guidance. The variable referral pattern to gastroenterology may have been due to lack of hepatology services in the Trust for a period of time. Furthermore, Fibroscan services only became available in the Trust in 2020 .

Ultrasound was overused and this may also be a reflection on previous lack of Fibroscan services. We feel that patients would benefit from a defined $\mathrm{HH}$ service and closer coordination of care between specialists.

\section{PWE-41 IS ABDOMINAL ULTRASONOGRAPHY A RELIABLE MODALITY TO DIAGNOSE COMPENSATED ADVANCED CHRONIC LIVER DISEASE?}

Hasaan Rafique*, Manushri Jain, Majid Khan, Sonika Sethi, Saleha Azhar, Chris Corbett, Debashis Haldar, Saqib Mumtaz. The Royal Wolverhampton NHS Trust, Wolverhampton, UK

\subsection{6/gutjnl-2021-BSG.231}

Introduction Compensated advanced chronic liver disease (cACLD) refers to a cohort of asymptomatic patients with advanced fibrosis who do not yet exhibit significant biochemical evidence of synthetic dysfunction or clinical and radiological manifestations of portal hypertension. Ultrasonography (US) is a first-line radiological investigation for patients with suspected chronic liver disease but its role in diagnosing cACLD is not well-established. The aim of this study is to assess the diagnostic accuracy of US in identifying patients with cACLD using categorical cut offs of Transient Elastography (TE) based on Baveno VI consensus.

Methods A retrospective cross-sectional observational study was conducted at the Royal Wolverhampton Hospital NHS Trust for patients with suspected chronic liver disease who underwent US, TE and screening blood tests within 12 months of each other as part of their investigations. Patients were included if; TE recorded a valid reading and the US report mentioned a radiological description suggestive of cirrhosis as per the nomenclature agreed with the local specialist sonographers (coarse echotexture, irregular liver margin, nodular or heterogeneous parenchyma, or cirrhosis). Patients were excluded if; US showed signs of portal hypertension, there was a past history of hepatic decompensation, liver transplant or heart failure, or if they were currently being evaluated for acute hepatitis. Sensitivity, specificity, positive and negative predictive value (PPV \& NPV) of US to detect cACLD were calculated using categorical cut offs of TE based on Baveno VI consensus (TE $>15 \mathrm{kPa}$ as positive and $<10 \mathrm{kPa}$ negative for cACLD respectively).

Results 1528 of 3357 screened patients were included; mean age 51 years, $64 \%$ men and $71 \%$ of white ethnicity. Aetiology of liver disease included [\%, n]; ALD (20, 312) NAFLD (29, 452), Hepatitis C $(11.4,175)$, Hepatitis B (11, 168), PBC (4, 63), AIH (2.7, 42), Haemochromatosis (2.7, 41), unspecified $(18,275)$.

The overall sensitivity and specificity of US for diagnosis of cACLD was $64.6 \%(\mathrm{p}<0.0001)$ and $78 \%(\mathrm{p}<0.0001)$ respectively, with PPV of $40 \% \quad(\mathrm{p}<0.0001)$ and NPV of $91 \%$ $(\mathrm{p}<0.0001)$. A similar trend was seen across all aetiologies.

We also found that a platelet count (PLT) of $<150 \mathrm{x}$ $10^{\wedge} 9 / \mathrm{L}$ and serum albumin concentration (ALB) of $<35 \mathrm{~g} / \mathrm{L}$ improved the PPV of US for detecting cACLD to $84 \%$ (95\% CI $73-95 \%, \mathrm{p}<0.0086$ ), while a normal PLT and ALB reduced the PPV to $21 \%$ (95\% CI $15.4-26.2 \%, \mathrm{p}<0.0001)$. Similarly, the NPV of US for excluding cACLD was $93 \%$ 
(95\% CI 91-94.7\%, p<0.0001) when both PLT and ALB were in normal range.

Conclusions US alone is not a reliable imaging modality for diagnosing cACLD. In suspected cases, TE should be carried out to determine the severity of hepatic fibrosis before a diagnosis of cACLD is made.

\section{PWE-42 MENTAL HEALTH READMISSIONS ARE INCREASED IN TRANSPLANT-FREE SURVIVORS OF ACUTE LIVER FAILURE}

${ }^{1}$ Mhairi C Donnelly*, ${ }^{2}$ Linda Williams, ${ }^{2}$ Nazir Lone, ${ }^{1}$ Kenneth J Simpson. ${ }^{1}$ Royal Infirmary of Edinburgh, Edinburgh, UK; ${ }^{2}$ University of Edinburgh, Edinburgh, UK

\subsection{6/gutjnl-2021-BSG.232}

Introduction Patients surviving acute liver failure (ALF) without transplantation (spontaneous survivors; SS) are at increased risk of later death and readmission to hospital. Most cases of ALF in Scotland are managed in the Scottish Liver Transplant Unit (SLTU) and are due to paracetamol overdose (POD). The aim of this work was to analyse mental health readmissions in Scottish ALF SS, compared with comparator cohorts.

Methods The index cohort (SLTU SS) consisted of patients admitted with severe acute liver injury or failure between 01/ $11 / 1992$ and 31/12/2014 who survived to hospital discharge without transplantation. Patients were identified from the SLTU ALF database. Control cohorts were: age, sex and postcode sector matched general population controls, patients admitted to hospital following POD in Scotland and not transferred to SLTU, patients admitted to Scottish intensive care units as non-surgical emergency admissions (SICSAG cohort) and patients surviving ALF with transplantation. Data related to mental health readmissions following discharge from the index admission were derived from the SMR04 database.

Results The SLTU SS cohort consisted of 708 patients $(80.2 \%$ POD). $25.6 \%$ of the SLTU SS had at least one mental health readmission $(29.5 \%$ of POD SS versus $10.0 \%$ of non-POD SS).

The SLTU SS were more likely to experience a mental health readmission compared with the matched control cohort (adjusted SHR 11.23, 95\% CI 8.55, 14.71), paracetamol overdose cohort (adjusted SHR 1.25, 95\%CI 1.04, 1.51) and the SICSAG cohort (adjusted SHR 2.76, 95\% CI 1.99, 3.82).

The adjusted relative rate of mental health readmissions was also higher in the SLTU SS compared with the matched control cohort $(10.63,95 \% 7.58,14.90)$, the paracetamol overdose cohort $(2.90,95 \%$ CI $2.23,3.77)$ and the SICSAG cohort $(5.91,95 \%$ CI $3.54,9.88)$. In the SLTU SS, POD aetiology and prior mental health admissions were predictors of later mental health readmissions.

The main psychiatric diagnosis in the SLTU SS was depressive disease (27.4\%) compared with alcohol misuse $(27.3 \%)$ in the matched controls, depressive disease (30.2\%) in the paracetamol overdose controls and alcohol misuse (32.1\%) in the SICSAG controls.

A comparative mental health morbidity analysis was not possible in the ALF survivors with transplant, as $<10$ posttransplant patients had any post-discharge mental health readmissions.

Conclusions Scottish ALF SS are at increased risk of mental health readmissions following discharge. The risk of readmission is higher in those with POD ALF compared with POD without ALF. Patients surviving ALF without transplant are currently discharged without follow up. This work suggests that psychiatric follow up -particularly in those with POD ALF - is warranted to reduce the burden of mental health readmissions.

\section{PWE-43 SARS-COV-2 ONE YEAR ON - THE WORRYING IMPACT ON EARLY DETECTION OF LIVER CANCERS}

1,2Daniel Geh*, 1,2Robyn Watson, 2,3Stuart McPherson, 2,35teven Masson, 2,3 Jessica Dyson, 2,3 Mhairi Donnelly, 2,3 Louise MacDougal, ${ }^{2,3}$ Preya Patel, ${ }^{3,4}$ John Hammond, ${ }^{3,5}$ Paul Turner, ${ }^{3,5}$ Tim Hoare, ${ }^{3,5}$ Kirsty Anderson, 3,5 Michael McNeil, ${ }^{3,4}$ Gourab Sen, ${ }^{3,4}$ Prof Steven White, ${ }^{3,4}$ Jeremy French, ${ }^{3,4}$ Stuart Robinson, Lucy Walker, ${ }^{1,2}$ Ms Misti McCain, ${ }^{6}$ Nick Wadd, ${ }^{7}$ Syed Asghar, ${ }^{3,8}$ Lavanya Mariappan, ${ }^{3,8}$ Jane Margetts, ${ }^{3}$ Yvonne Bury, ${ }^{3}$ Shreya Raman, ${ }^{3}$ Antony Darne, ${ }^{3}$ Beate Haugk, ${ }^{3,5}$ John Scott, ${ }^{3,5}$ Peter Littler, ${ }^{3,4}$ Derek M Manas, ${ }^{1,2,3}$ Helen L Reeves. 'Translational and Clinical Research institute, Newcastle University; ${ }^{2}$ Department of Medicine, Newcastle upon Tyne Hospitals NHS Foundation Trust; ${ }^{3}$ Hepatopancreatobiliary Multidisciplinary Team, Newcastle upon Tyne Hospitals NHS Foundation Trust; ${ }^{4}$ Department of Surgery, Newcastle upon Tyne Hospitals NHS Foundation Trust; ${ }^{5}$ Department of Radiology, Newcastle upon Tyne Hospitals NHS Foundation Trust; ${ }^{6}$ Department of Oncology, South Tees Hospital NHS Foundation Trust; ${ }^{7}$ Department of Oncology, Newcastle upon Tyne Hospitals NHS Foundation Trust; ${ }^{8}$ Department of Oncology, Cumberland Infirmary, North Cumbria Integrated Care NHS Foundation Trust

\subsection{6/gutjil-2021-BSG.233}

Background In Northern England new primary liver cancer (PLC) diagnoses have increased by $10-15 \%$ year on year. We evaluated the impact of the SARS-CoV-2 pandemic.

Method In this retrospective, observational, regional study we evaluated 455 new PLC patients referred to the Newcastle-
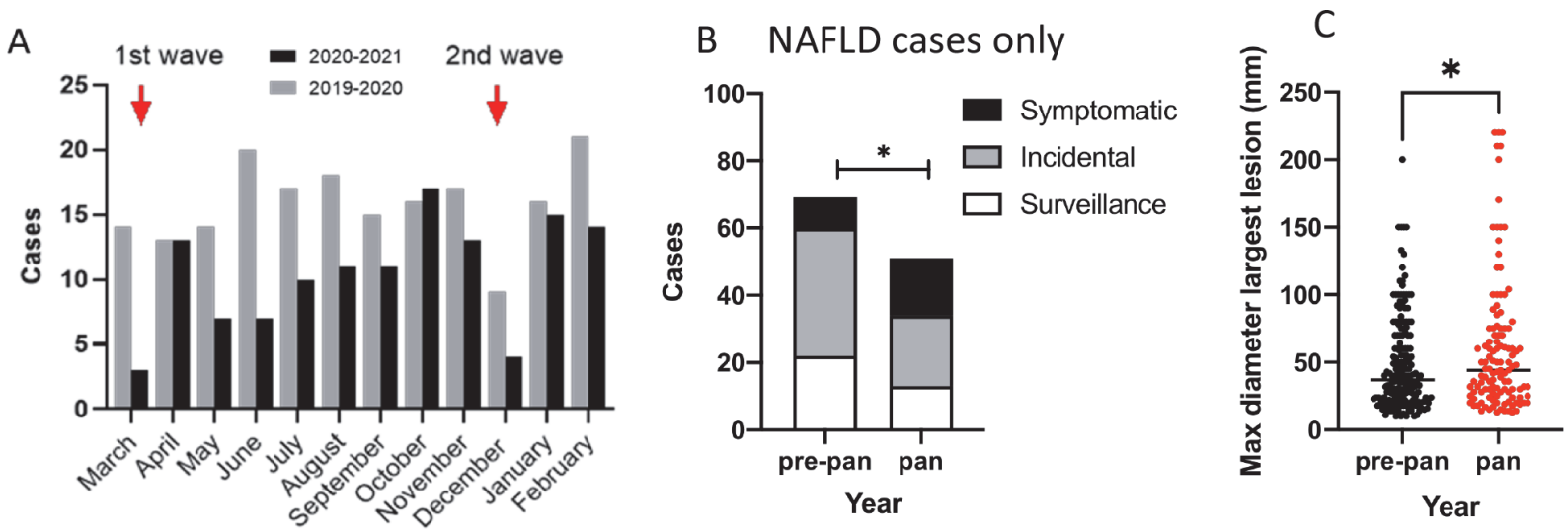

Abstract PWE-43 Figure 1 